\title{
CrimRxiv
}

\section{Anomie and collaboration in intelligence and operational police and border guard work in the Baltic Sea area: in-group mentality and construction of the other}

Goran Basic, Sophia Yakhlef

Published on: Jan 27, 2022

DOI: $10.21428 / \mathrm{cb} 6 \mathrm{ab} 371.2 \mathrm{e} 62 \mathrm{fccc}$

License: Creative Commons Attribution 4.0 International License (CC-BY 4.0). 
